\title{
Ageing, cortical injury and Alzheimer's disease-like pathology in the guinea pig brain.
}

Bates, K. A. ${ }^{a, b, c^{\wedge \star}}$, Vink R. ${ }^{d}$, Martins R. N. ${ }^{b, c, e}$ and Harvey A. R. ${ }^{a}$ a School of Anatomy, Physiology and Human Biology, The University of Western Australia. M309 35 Stirling Highway Crawley WA 6009, Australia.

b School of Psychiatry and Clinical Neuroscience, The University of Western Australia. M650 35 Stirling Highway Crawley WA 6009, Australia.

c The McCusker Foundation for Alzheimer's Disease Research Inc. Suite 22, Hollywood Medical Centre 85 Monash Ave, Nedlands WA 6009 Australia.

d School of Medical Sciences, University of Adelaide, Adelaide SA 5005, Australia. e School of Medical Sciences, Edith Cowan University. 270 Joondalup Drive Joondalup WA 6027, Australia.

${ }^{\wedge}$ Present address School of Animal Biology, University of Western Australia. M317 35 Stirling Highway Crawley, WA 6009, Australia.

Email addresses:

kristyn.bates@uwa.edu.au; Robert.Vink@adelaide.edu.au; r.martins@ecu.edu.au; alan.harvey@uwa.edu.au

${ }^{*}$ Corresponding Author

Telephone: +61086488 7502 Fax: +61 0864887527 


\section{Abstract}

Alzheimer's disease (AD) is a progressive neurodegenerative disorder characterised histopathologically by the abnormal deposition of the proteins amyloid-beta $(A \beta)$ and tau. A major issue for $A D$ research is the lack of an animal model that accurately replicates the human disease, thus making it difficult to investigate potential risk factors for AD such as head injury. Furthermore, as age remains the strongest risk factor for the majority of $A D$ cases, transgenic models in which mutant human genes are expressed throughout the lifespan of the animal provide only limited insight into age-related factors in disease development. Guinea pigs (Cavia porcellus) are of interest in $A D$ research because they have a similar $A \beta$ sequence to humans and thus may present a useful non-transgenic animal model of AD. Brains from guinea pigs aged 3 to 48 months were examined to determine the presence of ageassociated AD-like pathology. In addition, fluid percussion-induced brain injury was performed to characterise mechanisms underlying the association between AD risk and head injury. No statistically significant changes were detected in the overall response to ageing, although we did observe some region-specific changes. Diffuse deposits of $A \beta$ were found in the hippocampal region of the oldest animals and alterations in APP processing and tau immunoreactivity were observed with age. Brain injury resulted in a strong and sustained increase in APP and tau immunoreactivity without $A \beta$ deposition, over 7 days. Guinea pigs may therefore provide a useful model for investigating the influence of environmental and nongenetic risk factors on the pathogenesis of AD.

\section{$\underline{\text { Keywords }}$}

Alzheimer's disease, amyloid-beta, ageing, head injury. 


\section{$\underline{\text { Introduction }}$}

The most widely accepted hypothesis addressing the pathological basis of AD is the "amyloid hypothesis", which suggests that the altered metabolism of a small peptide, amyloid-beta $(A \beta)$, is the trigger for the pathogenic cascade that ultimately results in neuronal dysfunction and death. $A \beta$ is derived through sequential enzymatic cleavage of the amyloid precursor protein (APP), a type 1 integral membrane protein, by BACE ( $\beta$-site APP cleaving enzyme) and then $\gamma$-secretase, a multisubunit enzymatic complex. Age is the strongest risk factor that has been identified to date, yet it is still unclear as to why the clinical symptoms of $A D$ appear in later life when the neuropathology may begin many years, even decades, earlier (Villemagne et al. 2013).

A major hindrance to $A D$ research is the lack of an animal model that replicates all of the features of the human disease, namely a chronic, age-dependant neurodegenerative disease with characteristic AD histopathology. Transgenic animal models have yielded a number of important insights into disease mechanisms and are a useful tool for therapeutic design and evaluation. However, the genetic manipulation necessary to produce them does not accurately reflect the human disease state, and accordingly limits at least some of the conclusions that can be drawn.

An alternative strategy may be to investigate potential risk factors in species with similar $A \beta$ sequence to humans. One candidate is the guinea pig (Beck et al. 1997). In vitro studies using guinea pig neuronal cells have demonstrated that APP processing in this species is identical to that in humans (Beck et al. 2000; Beck et al. 2003). Guinea pigs have been used in a small number of studies to investigate the 
effect of glutamate (Stephenson and Clemens 1998), serotonin (Arjona et al. 2002), estrogen (Petanceska et al. 2000), testosterone (Wahjoepramono et al. 2008; Wahjoepramono et al. 2011), $\beta$-secretase inhibitors (Hook et al. 2007; Jeppsson et al. 2012) and cholesterol (Fassbender et al. 2001) on $A \beta$ production. Further, the effect of PKC (Rossner et al. 2000) and $\mathrm{GABA}_{\mathrm{A}}$ receptor modulation (Marcade et al. 2008) on APP processing has also been investigated in this species.

Traumatic brain injury (TBI) has been identified as a risk factor for AD (Bachman et al. 2003; Fleminger et al. 2003). Following TBI, CSF levels of tau (Franz et al. 2003), APP and A $\beta$ increase (Olsson et al. 2004) and post mortem analyses of brains from TBI patients show widespread distribution of $A B$ containing plaques and other $A D$ like pathology including gliosis (Gentleman et al. 1993; Griffin et al. 1994; Horsburgh et al. 2000; Ikonomovic et al. 2004). In mice (Iwata et al. 2002), rats (Blasko et al. 2004) and sheep (Van den Heuvel et al. 1999), APP mRNA and protein expression rapidly, albeit transiently, increase in response to cortical injury.

To date, no studies have investigated the relationship between ageing and AD-like pathology in a non-primate species with human-like APP. Similarly, head injury models have not been performed in animals with similar $A \beta$ sequence to humans. Therefore, a series of pilot studies was conducted to investigate the suitability of the guinea pig as a non-transgenic animal model for AD. A longitudinal ageing study and a fluid percussion-induced brain injury (FPI) study were conducted to determine whether any AD-like pathology could be detected in the guinea pig brain.

\section{Methods}


All animal experimentation was approved by the Animal Ethics Committees of The University of Western Australia and the Institute of Medical and Veterinary Sciences and carried out under published National Health and Medical Research Council guidelines. Tissue collection was performed after a lethal dose of sodium pentobarbitone followed by transcardial perfusion with fixative $(10 \%$ buffered formal saline) or by harvesting fresh unfixed tissue snap frozen in liquid nitrogen. Tissue embedding and preparation for histochemical and western blotting procedures were performed as previously described (Bates et al. 2002; Bates et al. 2007).

The following antibodies were used to investigate APP and A $\beta$ metabolism: WO2 (gift from Professor Colin Masters, University of Melbourne, VIC Australia, 1/10 for histochemistry, 1/100 for western blots), anti-BACE1 polyclonal antibody (Thermo Scientific, IL USA, 1/100 for histochemistry) and anti-nicastrin polyclonal antibody (Nct, Invitrogen CA USA, 1/100 for histochemistry). In addition, tau was investigated using a monoclonal Tau-5 antibody (Invitrogen CA USA, 1/1000 for histochemistry, 1/5000 for western blots). A modified Congo Red stain (Bancroft and Stevens 1982) was used to detect the presence of fibrillar $A \beta$ deposits. Astrocyte reactivity was investigated using monoclonal anti-GFAP antibody (Sigma MO USA, 1/400 for histochemistry, 1/5000 for western blots).

\section{Ageing study}

Animals $3,6,9,12,18,24,36$ and 48 months of age of both sexes were used. Each age group had 7 animals, 3 were processed for histological examination and the remaining 4 brains were dissected into 10 brain regions; frontal, parietal, occipital and temporal cortices, cerebellum, hippocampus, thalamus, midbrain, brainstem and basal ganglia for western and ELISA analysis (see below). 


\section{Fluid percussion injury study}

Animals were fasted (with access to water) 12 hours prior to surgery to assist in recovery from anaesthesia. Anaesthesia was achieved as follows; $0.1 \mathrm{ml}$ atropine sulfate $(0.6 \mathrm{mg} / \mathrm{ml})$ was administered subcutaneously. After 5 minutes, diazepam (5 $\mathrm{mg} / \mathrm{kg}$ i.p., Parnell Laboratories, Australia) was given i.p. at a dose of $5 \mathrm{mg} / \mathrm{kg}$ body weight. Once the animal was sufficiently sedated (approximately 20 minutes post diazepam injection) hypnorm was given intramuscularly in the rear limb (VetaPharm, $0.315 \mathrm{mg} / \mathrm{ml}$ fentanyl citrate and $10 \mathrm{mg} / \mathrm{ml}$ fluanisone, $1 \mathrm{ml} / \mathrm{kg}$ i.m.).

A mid-sagittal incision was made and a $5 \mathrm{~mm}$ craniotomy performed centred over the left parietal cortex (Dudas et al. 2000) to facilitate induction of lateral fluid percussion injury (FPI) as previously described (Vink et al. 1988). The dura was left intact and a female Leur-loc was placed over the craniotomy and secured with dental cement. The fluid percussion device consisted of a plexiglass cylindrical reservoir, $60 \mathrm{~cm}$ long and $4.5 \mathrm{~cm}$ in diameter, bounded at one end by a plexiglass, rubber-covered piston mounted on O-rings. The opposite end was fitted with a $2 \mathrm{~cm}$ long metal housing on which a pressure transducer was mounted and connected to a $5 \mathrm{~mm}$ tube $(2 \mathrm{~mm}$ inner diameter) that terminated with a male leur-loc fitting. The whole system was filled with physiological saline at room temperature.

At the time of injury the male leur-loc tube was connected securely to the female leur-loc on the skull. To produce an insult, a $4.8 \mathrm{~kg}$ pendulum was dropped from a pre-determined height to impound the rubber-covered piston, producing a pressure pulse of $2.1 \pm 0.2$ atmospheres. We have previously shown that this equates to a mild to moderate injury in rats (Vink, Faden et al. 1988). Injury resulted in apnoea so animals were manually resuscitated for a period ranging from 2 to 15 minutes. The 
scalp was then closed with surgical staples. One dose of post-operative analgesia (0.16mg/kg buprenorphine i.p.) along with $1 \mathrm{ml}$ sterile saline/hour for 4 hours was administered. Sham animals underwent the same procedures without the FPI. From the moment of sedation to recovery, animal temperature was maintained throughout using a thermostatically controlled heating blanket.

\section{Protein detection and analysis: Western blotting and ELISA}

The method for tissue preparation and western blot analysis has been described previously (Martins et al. 2001; Bates, Fonte et al. 2002; Bates, Martins et al. 2007). Protein concentration in the sample homogenate was calculated using the Micro BCA protein assay kit (Pierce IL, USA). Samples containing $25 \mu \mathrm{g}$ of total protein was resuspended in sample buffer $(166 \mathrm{mM}$ Tris- $\mathrm{HCl}, 8 \%$ SDS, $0.53 \mathrm{M}$ glycine, $5 \% \beta$ mercaptoethanol, $\mathrm{pH}$ 6.8). Samples were arranged such that each gel contained samples from each age group for a particular brain region. An internal control sample was created by pooling cerebellum homogenates from different ages and this was run on each gel to account for gel-to-gel variations. Cerebellum was chosen because this was the easiest brain region to distinguish, is usually spared from AD-like pathology in human disease, and yielded more sample than required for the experiments.

After electrophoretic separation and transfer of proteins, the nitrocellulose membranes were cut into sections corresponding to specific molecular weights to enable probing for different proteins from the same blot. Membranes were probed for APP and A , C-100 and tau. Samples from each age group were normalised against the internal control. Due to the nature of the comparisons to be made, protein levels were measured and age-related effects were constructed by 
calculating the fold change in protein levels using 3 month-old samples as a baseline reading.

Immunohistochemistry

The protocol for immunohistochemical detection of paraffin embedded sections has been published previously (Harvey et al. 1997; Martins, Taddei et al. 2001; Bates, Fonte et al. 2002; Bates, Martins et al. 2007). Briefly, sections were de-waxed and rehydrated through decreasing concentrations of ethanol back to water. Antigen retrieval was performed by boiling in $10 \mathrm{mM}$ citrate buffer ( $\mathrm{pH}$ 6) followed by treatment with $80 \mathrm{mM}$ ammonium chloride for 10 minutes. Endogenous peroxidases were quenched by treating the sections with $0.06 \% \mathrm{H}_{2} \mathrm{O}_{2}$ for 20 minutes. The sections were then washed in PBS and incubated with the appropriate antibody in diluent $\left(0.2 \%\right.$ Triton $\mathrm{X}-100,1 \% \mathrm{BSA}$ in PBS) at $4^{\circ} \mathrm{C}$ overnight. The next day, the sections were washed in PBS before being incubated with the correct biotinylated secondary antibody diluted 1:800 in the PBS diluent for 2 hours at room temperature. After the sections had been washed, they were incubated with the Vectastain $A B C$ kit as per manufacturer instructions. The sections were washed and reacted with DAB reagent (diaminobenzidine, Pierce) until sufficient colour development was achieved.

Image analysis and quantification

Images of immunohistochemical stains were taken using a CoolSnap camera attached to a Nikon Eclipse E800 microscope. Films from western blot experiments were scanned on a BioRad GS-800 densitometer and band analysed using Quantity One software (BioRad, CA USA). For each protein of interest, the same exposure time was used and only non-saturated bands were used in the analysis. Relative 
optical density was calculated and arranged in Microsoft Excel and the data were analysed by one-way ANOVA using SPSS software (SPSS version 15.0 for Windows), with significance set at $\leq 0.05$. Because of the high degree of variability between samples, the data for age groups are pooled for ease of interpretation.

\section{$\underline{\text { Results }}$}

Effect of ageing on APP metabolism in the guinea pig.

Perhaps due to the large degree of variability between samples, we were unable to detect any overall statistically significant changes associated with ageing in the proteins of interest, however a number of intriguing and potentially important trends were seen. APP immunoreactivity was stronger in some, but not all brain regions from aged animals (48 months) compared to young, 3 month-old animals. Brain regions with increased APP staining in aged animals were the frontal cortex, midbrain and hippocampus. No age-related changes in APP staining were seen in other brain regions.

Dense $A \beta$ deposits, as defined by Congo red and PAS reactivity, were not observed at any time point in any of the brain regions examined. However, diffuse $A \beta$ deposits were seen using WO2 antibody in the CA1 hippocampal region from aged (48 month-old) animals (Figure 1a, b). No glial cell reactivity was seen surrounding these deposits, further suggesting they were diffuse in nature.

Semi-quantitative analysis by western blot confirmed these observations. The fold change in APP levels with age was calculated across the brain regions studied (Figure 1c and d). Considerable variation was observed in the frontal and temporal 
cortices and midbrain across all time points, and some interesting trends were observed in terms of regional sensitivities to age. Fold change in APP levels was most pronounced in the frontal and temporal cortices and in the hippocampus, midbrain and brainstem. Little change was observed in other brain regions. $A \beta$ levels in total homogenate from all brain regions was assessed by western blot and ELISA, however the levels were below the detection limit of these techniques (approximately $20 \mathrm{pg}$ for westerns and 10pg/ml for ELISA).

The effect of age on APP processing to favour the amyloidogenic pathway was assessed by semi-quantitative analysis of PS-1 and C-100 levels by western blot and BACE-1 immunoreactivity. The fold-change in C-100 levels was measured as an indicator of amyloidogenic processing of APP. To further explore an age effect on amyloidogenic processing, the ratio of C-100 levels to APP was calculated. Those regions with a ratio close to one would indicate that any increase in C-100 might simply be due to elevated APP levels. Conversely, a ratio greater than one may indicate that amyloidogenic processing is occurring preferentially to nonamyloidogenic APP processing. A ratio greater than one was most consistently seen in the temporal and cerebellar cortices and in the brainstem and basal ganglia (Figure 2). The ratio increased with age in the frontal and temporal cortices and the cerebellum and basal ganglia. Lastly, another indicator of amyloidogenic processing, BACE-1 reactivity was examined by immunohistochemistry. BACE-1 immunoreactivity increased with age in the temporal (Figure $3 b$ ) and frontal cortex (not shown) in the hippocampus and basal ganglia (not shown).

Age-related effects on tau pathology 
Tau staining was observed as cytoplasmic staining surrounding the nucleus creating a halo-like effect, and was similar across ages in some brain regions including the parietal cortex. However, in frontal cortex (Figure 3c and d), hippocampus and thalamus (not shown), tau staining was elevated in tissue from 48-month old animals. These results were confirmed by semi-quantitative analysis (Figure 3e). Tau levels increased slightly (albeit not significantly) with age in cortical regions, with larger increases in the occipital cortex and no changes observed in other brain regions of interest (data not shown). Multiple bands were detected in 48-month samples from a number of brain regions including occipital cortex and hippocampus, which may indicate an age-related alteration in tau metabolism.

\section{Effect of FPI on APP metabolism in the guinea pig}

The injury to guinea pigs was more severe than expected for this pulse pressure (Vink, Faden et al. 1988) with approximately 95\% of animals requiring manual resuscitation for a period ranging from 2 to 15 minutes immediately after the injury. Post mortem examination revealed extensive sub-arachnoid haemorrhaging, particularly around the brainstem region. Table 1 gives a qualitative summary of the events observed over time as a result of FPI. Representative micrographs of APP immunoreactivity ipsilateral and contralateral to, and at various time points after, the injury are shown in Figure 4. APP immunoreactivity was comparatively greater in the ipsilateral cortex and hippocampus 24 hours post-injury (Figure $4 a$ and e). APP staining was intense in both axons and cell bodies. This pattern or reactivity was sustained in ipsilateral cortex and hippocampus up to 7 days post-injury (Figure 4c and $\mathrm{g}$ ). $\mathrm{A} \beta$ deposition was not detected at any time point post-injury. 
To assess APP secretase activity, BACE-1 ( $\beta$-secretase) and Nct (a component of the putative $\gamma$-secretase) immunoreactivity was assessed. BACE- 1 immunoreactivity was increased at early time points, but had reverted to normal levels by 7 days postinjury. Axonal staining in injured areas was punctate compared to the smooth axonal staining observed on the non-injured side (not shown). Nicastrin (Nct) staining was also increased as a consequence of FPI in a time-course similar to BACE-1. Nct immunoreactivity was strongest in the injured cortex at 24 hours. Nct staining at other time points and contralateral to the injury was similar to that observed in controls (not shown).

\section{Effect of FPI on tau metabolism in the guinea pig}

Tau staining was increased in cell bodies of injured neurons (Figure 5a) at all time points post-injury compared to sham controls and contralateral cortex. Tau reactivity was also strong in ipsilateral hippocampus at all time points, particularly 3 days postFPI, and increased steadily in contralateral hippocampus up to 7 days post-FPI (Figure 5b).

\section{Discussion}

An animal model that produces all the pathological features of the human AD brain has not been developed. Animals with human-like endogenous $A \beta$ could provide valuable insights into the full spectrum of AD pathology. The guinea pig is one such animal, and a small number of studies have investigated factors that contribute to and modulate APP processing in this species. The aim of this study was to assess 
the suitability of the guinea pig as a model to investigate two risk factors for $A D$, namely ageing and head injury.

Ageing and $A D$-associated proteins in the guinea pig brain

Age-associated trends for a number of AD-related proteins were seen in the guinea pig brain. Age may influence the processing of APP to favour amyloidogenic processing as suggested by the increase in C-100 and BACE-1 observed in this study. Although quantitative measures of $A \beta$ could not be obtained, diffuse $A \beta$ deposition was observed in the hippocampal formation of the oldest animals used in this study (48 months of age). These data suggest that APP and A $\beta$-associated pathology may develop with age. The oldest age of animals that were used in this study was 4 years of age (48 months) whereas the average life span of the guinea pig is 5-7 years, with some reports of animals reaching 8-9 years of age. As such, four years of age may be equivalent to a 50-60 year old human. Samples from older animals may have a more pronounced and overt AD-like pathology.

Alternatively, $A \beta$ clearance mechanisms in the guinea pig may be more efficient than those in the human. This explanation may offer exciting new possibilities in deciphering the mechanisms of $A \beta$ clearance and improving therapeutic design. Interestingly, cholesterol metabolism in the guinea pig is very similar to the human, making them an appropriate model for human cholesterol and lipoprotein metabolism (Fernandez 2001). In addition to lipoprotein (i.e. apolipoprotein E) associated effects, the levels or activities of $A \beta$ degrading enzymes (i.e. neprilysin and insulindegrading enzyme) may be different in this species.

Analysis of tau protein suggests a possible age effect on tau levels. This effect was most pronounced in the frontal and occipital cortices, and in the hippocampus. 
Further investigation and validation of the effect seen here may reveal how agerelated processes modulate tau hyperphosphorylation and cytoskeleton instability.

A standout feature in the data presented here is that some brain regions in the guinea pig appeared to be more susceptible to age-related protein change than others. Regions particularly vulnerable to AD-associated change include the hippocampus, and the frontal and temporal cortices, a striking similarity to the regional vulnerability evident in the human disease (Braak and Braak 1997). Interestingly, the fold changes in non-cortical regions were large in comparison to cortical regions. This was an unexpected finding, though it is important to note that numerous studies have reported the presence of sub-cortical pathology in $A D$ (for example (Braak et al. 2011; Attems et al. 2012)). The guinea pig may yield insights into the underlying mechanisms of this regional variability.

$F P l$ in the guinea pig as a model for $A D$

The histopathology observed in the injured guinea pig brain lends support to the hypothesis that closed head injury can result in AD-like changes. FPI resulted in a time-dependent change in the levels of a number of proteins implicated in $A D$ pathogenesis, including APP, BACE-1 and tau. A $\beta$ deposition was not observed in the current study however FPI increased APP expression in cell bodies and axons at the injury site. In addition, the increased levels of BACE-1 and nicastrin may indicate altered APP metabolism to favour A $\beta$ production in response to FPI (Nadler et al. 2008). Tau reactivity was also increased as a result of FPI, suggesting that the neuronal cytoskeletons are damaged.

The present study involved injuring the parietal cortex in the guinea pig. FPI elicited a strong pathological response in the underlying hippocampus, yet not in any other 
brain region studied. The hippocampal formation has been previously identified as a particularly vulnerable region to traumatic events (Bernaudin et al. 1998; Mclntosh et al. 1998; Sato et al. 2001; Tong et al. 2002), ageing (Adams et al. 2001; Adams et al. 2002; Gallagher 2003) and neurodegenerative processes in general (Arriagada et al. 1992; Braak and Braak 1997; Bastianetto et al. 1999). The underlying mechanisms behind this selective vulnerability remain to be elucidated.

The conclusions that can be drawn from this study are confined by a number of factors. The study was limited to a small number of animals due to difficulties in obtaining guinea pigs and the animals used in this study were ex-breeders of both sexes, sourced from two locations, thus providing a number of confounding variables such as sex, breeding effects, diet and housing. It should also be noted that a degree of variability would be expected in out-bred animals, such as those used in this study. Increasing the sample size in order to reduce these confounding variables could thus be of value. Nonetheless the data presented here suggest that the guinea pig may indeed prove useful in investigating environmental influences on $A D$ pathogenesis, consistent with other guinea pig studies from our laboratory (Wahjoepramono, Wijaya et al. 2008; Wahjoepramono, Wijaya et al. 2011). Because guinea pig $A \beta$ is similar to human, injury studies in this species may be more applicable to the pathobiology of human AD. Such guinea pig models would be particularly relevant to late onset, sporadic $A D$, which is by far the most common form of $A D$ and is associated with a wide variety of risk factors.

\section{$\underline{\text { Acknowledgements }}$}

KAB was supported by an Australian Postgraduate Award and a Jean Rogerson Postgraduate Scholarship, and receives funding from the Neurotrauma Research 
Program of Western Australia (NRP). RNM receives support from The McCusker Foundation for Alzheimer's Disease Research Inc., NHMRC, CSIRO and Edith Cowan University. ARH receives funding from the NHMRC, ARC and NRP.

\section{Disclosure statement}

The authors have no conflicts of interest and all authors approve this submission. The manuscript has not been submitted for publication previously. KAB receives funding from the Neurotrauma Research Program (NRP). All other authors receive funding as listed under acknowledgements. Animal ethics approval was granted for the experiments. 


\section{$\underline{\text { References }}$}

Adams, M. M., S. E. Fink, R. A. Shah, W. G. Janssen, S. Hayashi, T. A. Milner, B. S. McEwen and J. H. Morrison, 2002. Estrogen and aging affect the subcellular distribution of estrogen receptor-alpha in the hippocampus of female rats. $J$ Neurosci 22, 3608-3614.

Adams, M. M., R. A. Shah, W. G. Janssen and J. H. Morrison, 2001. Different modes of hippocampal plasticity in response to estrogen in young and aged female rats. Proc Natl Acad Sci U S A 98, 8071-8076.

Arjona, A. A., A. M. Pooler, R. K. Lee and R. J. Wurtman, 2002. Effect of a 5-HT(2C) serotonin agonist, dexnorfenfluramine, on amyloid precursor protein metabolism in guinea pigs. Brain Res 951, 135-140.

Arriagada, P. V., J. H. Growdon, E. T. Hedley-Whyte and B. T. Hyman, 1992. Neurofibrillary tangles but not senile plaques parallel duration and severity of Alzheimer's disease. Neurology 42, 631-639.

Attems, J., D. R. Thal and K. A. Jellinger, 2012. The relationship between subcortical tau pathology and Alzheimer's disease. Biochem Soc Trans 40, 711-715.

Bachman, D. L., R. C. Green, K. S. Benke, L. A. Cupples and L. A. Farrer, 2003. Comparison of Alzheimer's disease risk factors in white and African American families. Neurology 60, 1372-1374.

Bancroft, J. D. and A. Stevens, 1982. Theory and Practise of Histological Techniques. London, Churchill-Livingstone.

Bastianetto, S., C. Ramassamy, J. Poirier and R. Quirion, 1999. Dehydroepiandrosterone (DHEA) protects hippocampal cells from oxidative stress-induced damage. Brain Res Mol Brain Res 66, 35-41. 
Bates, K. A., J. Fonte, T. A. Robertson, R. N. Martins and A. R. Harvey, 2002. Chronic gliosis triggers Alzheimer's disease-like processing of amyloid precursor protein. Neuroscience 113, 785-796.

Bates, K. A., R. N. Martins and A. R. Harvey, 2007. Oxidative stress in a rat model of chronic gliosis. Neurobiol Aging 28, 995-1008.

Beck, M., V. Bigl and S. Rossner, 2003. Guinea pigs as a nontransgenic model for APP processing in vitro and in vivo. Neurochem Res 28, 637-644.

Beck, M., M. K. Bruckner, M. Holzer, S. Kaap, T. Pannicke, T. Arendt and V. Bigl, 2000. Guinea-pig primary cell cultures provide a model to study expression and amyloidogenic processing of endogenous amyloid precursor protein. Neuroscience 95, 243-254.

Beck, M., D. Muller and V. Bigl, 1997. Amyloid precursor protein in guinea pigs-complete cDNA sequence and alternative splicing. Biochim Biophys Acta $1351,17-21$.

Bernaudin, M., A. Nouvelot, E. T. MacKenzie and E. Petit, 1998. Selective neuronal vulnerability and specific glial reactions in hippocampal and neocortical organotypic cultures submitted to ischemia. Exp Neurol 150, 30-39.

Blasko, I., R. Beer, M. Bigl, J. Apelt, G. Franz, D. Rudzki, G. Ransmayr, A. Kampfl and R. Schliebs, 2004. Experimental traumatic brain injury in rats stimulates the expression, production and activity of Alzheimer's disease beta-secretase (BACE-1). J Neural Transm 111, 523-536.

Braak, H. and E. Braak, 1997. Frequency of stages of Alzheimer-related lesions in different age categories. Neurobiol Aging 18, 351-357. 
Braak, H., D. R. Thal, E. Ghebremedhin and K. Del Tredici, 2011. Stages of the pathologic process in Alzheimer disease: age categories from 1 to 100 years. J Neuropathol Exp Neurol 70, 960-969.

Dudas, B., A. Mihaly and I. Hanin, 2000. A ventral approach to stereotaxy of the guinea pig brain. J Neurosci Methods 99, 79-83.

Fassbender, K., M. Simons, C. Bergmann, M. Stroick, D. Lutjohann, P. Keller, H. Runz, S. Kuhl, T. Bertsch, K. von Bergmann, M. Hennerici, K. Beyreuther and T. Hartmann, 2001. Simvastatin strongly reduces levels of Alzheimer's disease beta - amyloid peptides Abeta 42 and Abeta 40 in vitro and in vivo. Proc Natl Acad Sci U S A 98, 5856-5861.

Fernandez, M. L., 2001. Guinea pigs as models for cholesterol and lipoprotein metabolism. J Nutr 131, 10-20.

Fleminger, S., D. L. Oliver, S. Lovestone, S. Rabe-Hesketh and A. Giora, 2003. Head injury as a risk factor for Alzheimer's disease: the evidence 10 years on; a partial replication. J Neurol Neurosurg Psychiatry 74, 857-862.

Franz, G., R. Beer, A. Kampfl, K. Engelhardt, E. Schmutzhard, H. Ulmer and F. Deisenhammer, 2003. Amyloid beta 1-42 and tau in cerebrospinal fluid after severe traumatic brain injury. Neurology 60, 1457-1461.

Gallagher, M., 2003. Aging and hippocampal/cortical circuits in rodents. Alzheimer Dis Assoc Disord 17 Suppl 2, S45-47.

Gentleman, S. M., D. I. Graham and G. W. Roberts, 1993. Molecular pathology of head trauma: altered beta APP metabolism and the aetiology of Alzheimer's disease. Prog Brain Res 96, 237-246.

Griffin, W. S., J. G. Sheng, S. M. Gentleman, D. I. Graham, R. E. Mrak and G. W. Roberts, 1994. Microglial interleukin-1 alpha expression in human head injury: 
correlations with neuronal and neuritic beta-amyloid precursor protein expression. Neurosci Lett 176, 133-136.

Harvey, A. R., C. L. Kendall and E. Sykova, 1997. The status and organization of astrocytes, oligodendroglia and microglia in grafts of fetal rat cerebral cortex. Neurosci Lett 228, 58-62.

Hook, G., V. Y. Hook and M. Kindy, 2007. Cysteine protease inhibitors reduce brain beta-amyloid and beta-secretase activity in vivo and are potential Alzheimer's disease therapeutics. Biol Chem 388, 979-983.

Horsburgh, K., G. M. Cole, F. Yang, M. J. Savage, B. D. Greenberg, S. M. Gentleman, D. I. Graham and J. A. Nicoll, 2000. beta-amyloid (Abeta)42(43), abeta42, abeta40 and apoE immunostaining of plaques in fatal head injury. Neuropathol Appl Neurobiol 26, 124-132.

Ikonomovic, M. D., K. Uryu, E. E. Abrahamson, J. R. Ciallella, J. Q. Trojanowski, V. M. Lee, R. S. Clark, D. W. Marion, S. R. Wisniewski and S. T. DeKosky, 2004. Alzheimer's pathology in human temporal cortex surgically excised after severe brain injury. Exp Neurol 190, 192-203.

Iwata, A., X. H. Chen, T. K. McIntosh, K. D. Browne and D. H. Smith, 2002. Longterm accumulation of amyloid-beta in axons following brain trauma without persistent upregulation of amyloid precursor protein genes. J Neuropathol Exp Neurol 61, 1056-1068.

Jeppsson, F., S. Eketjall, J. Janson, S. Karlstrom, S. Gustavsson, L. L. Olsson, A. C. Radesater, B. Ploeger, G. Cebers, K. Kolmodin, B. M. Swahn, S. von Berg, T. Bueters and J. Falting, 2012. Discovery of AZD3839, a potent and selective BACE1 inhibitor clinical candidate for the treatment of Alzheimer disease. $J$ Biol Chem 287, 41245-41257. 
Marcade, M., J. Bourdin, N. Loiseau, H. Peillon, A. Rayer, D. Drouin, F. Schweighoffer and L. Desire, 2008. Etazolate, a neuroprotective drug linking $\operatorname{GABA}(\mathrm{A})$ receptor pharmacology to amyloid precursor protein processing. $\mathrm{J}$ Neurochem 106, 392-404.

Martins, R. N., K. Taddei, C. Kendall, G. Evin, K. A. Bates and A. R. Harvey, 2001. Altered expression of apolipoprotein E, amyloid precursor protein and presenilin-1 is associated with chronic reactive gliosis in rat cortical tissue. Neuroscience 106, 557-569.

McIntosh, T. K., K. E. Saatman, R. Raghupathi, D. I. Graham, D. H. Smith, V. M. Lee and J. Q. Trojanowski, 1998. The Dorothy Russell Memorial Lecture. The molecular and cellular sequelae of experimental traumatic brain injury: pathogenetic mechanisms. Neuropathol Appl Neurobiol 24, 251-267.

Nadler, Y., A. Alexandrovich, N. Grigoriadis, T. Hartmann, K. S. Rao, E. Shohami and R. Stein, 2008. Increased expression of the gamma-secretase components presenilin-1 and nicastrin in activated astrocytes and microglia following traumatic brain injury. Glia 56, 552-567.

Olsson, A., L. Csajbok, M. Ost, K. Hoglund, K. Nylen, L. Rosengren, B. Nellgard and K. Blennow, 2004. Marked increase of beta-amyloid(1-42) and amyloid precursor protein in ventricular cerebrospinal fluid after severe traumatic brain injury. J Neurol 251, 870-876.

Petanceska, S. S., V. Nagy, D. Frail and S. Gandy, 2000. Ovariectomy and 17betaestradiol modulate the levels of Alzheimer's amyloid beta peptides in brain. Exp Gerontol 35, 1317-1325.

Rossner, S., M. Beck, T. Stahl, K. Mendla, R. Schliebs and V. Bigl, 2000. Constitutive overactivation of protein kinase $\mathrm{C}$ in guinea pig brain increases 
alpha-secretory APP processing without decreasing beta- amyloid generation. Eur J Neurosci 12, 3191-3200.

Sato, M., E. Chang, T. Igarashi and L. J. Noble, 2001. Neuronal injury and loss after traumatic brain injury: time course and regional variability. Brain Research. $917,45-54$.

Stephenson, D. T. and J. A. Clemens, 1998. Metabotropic glutamate receptor activation in vivo induces intraneuronal amyloid immunoreactivity in guinea pig hippocampus. Neurochem Int 33, 83-93.

Tong, W., T. Igarashi, D. M. Ferriero and L. J. Noble, 2002. Traumatic brain injury in the immature mouse brain: characterization of regional vulnerability. Experimental Neurology. 176, 105-116.

Van den Heuvel, C., P. C. Blumbergs, J. W. Finnie, J. Manavis, N. R. Jones, P. L. Reilly and R. A. Pereira, 1999. Upregulation of amyloid precursor protein messenger RNA in response to traumatic brain injury: an ovine head impact model. Exp Neurol 159, 441-450.

Villemagne, V. L., S. Burnham, P. Bourgeat, B. Brown, K. A. Ellis, O. Salvado, C. Szoeke, S. L. Macaulay, R. Martins, P. Maruff, D. Ames, C. C. Rowe and C. L. Masters, 2013. Amyloid beta deposition, neurodegeneration, and cognitive decline in sporadic Alzheimer's disease: a prospective cohort study. Lancet Neurol

Vink, R., A. I. Faden and T. K. Mclntosh, 1988. Changes in cellular bioenergetic state following graded traumatic brain injury in rats: determination by phosphorus 31 magnetic resonance spectroscopy. J Neurotrauma 5, 315-330. Wahjoepramono, E. J., L. K. Wijaya, K. Taddei, K. A. Bates, M. Howard, G. Martins, K. deRuyck, P. M. Matthews, G. Verdile and R. N. Martins, 2011. Direct 
exposure of guinea pig CNS to human luteinizing hormone increases cerebrospinal fluid and cerebral beta amyloid levels. Neuroendocrinology 94, 313-322.

Wahjoepramono, E. J., L. K. Wijaya, K. Taddei, G. Martins, M. Howard, K. de Ruyck, K. Bates, S. S. Dhaliwal, G. Verdile, M. Carruthers and R. N. Martins, 2008. Distinct effects of testosterone on plasma and cerebrospinal fluid amyloidbeta levels. J Alzheimers Dis 15, 129-137. 
Figure 1. Analysis of APP levels in the ageing guinea pig brain. APP immunohistochemistry was performed using the antibody WO2 on brain sections from animals of all age groups. Diffuse $A \beta$ deposits were observed in hippocampi from 48 month old animals ( $a$ and $b$, arrows). Scale bar $=100 \mu m$. APP levels were analysed semi-quantitatively using western blotting techniques. The fold change in APP levels was averaged for the number of animals $(n=4)$ across each age group $(n=8)(c, d)$. APP levels may be elevated in frontal and temporal cortices, despite the large error bars, (c, representative blot for temporal cortex shown, with position of molecular weight marker, 99kDa) and in midbrain, hippocampus and brainstem (d, representative blot for hippocampus shown, with position of molecular weight marker) with age. Data are presented as fold change +/- SEM.

Figure 2. Change in the ratio of $\mathrm{C}-100$ to APP in brain regions as a function of age (time). The ratio of $\mathrm{C}-100$ to APP produced was calculated to determine if APP metabolism is altered with age in the guinea pig. The ratio in cortical regions is given in (a) and in other brain regions studied in (b). Age may influence amyloidogenic processing in the frontal, temporal and cerebellar cortices, and particularly in the basal ganglia.

Figure 3. Effect of age on BACE-1 and Tau reactivity in various brain regions. BACE-1 and Tau staining was compared in all brain regions across all age groups studied. In the temporal cortex, BACE-1 levels were increased in 48 (b) compared to 3 month-old animals (a). Staining was strong in cell bodies and axons (black arrows). Tau staining was increased in the frontal cortex (d) in 48 month old animals compared to 3 month-old animals (c). Some cells had a homogenous staining pattern, perhaps indicative of tau hyperphosphorylation (black arrows, d). An example of staining seen without the primary antibody is shown (e), scale bar= 
$100 \mu \mathrm{m}$. Tau levels were assessed semi-quantitatively by western blot analysis (f). A representative blot from the occipital cortex is shown, along with the position of the molecular weight marker $(69 \mathrm{kDa})$. The presence of multiple bands may indicate an age-related effect on tau metabolism. The fold change in tau levels was averaged for the number of animals $(n=4)$ across each group $(n=8)$ and tau levels may increase with time in most cortical regions. Data are presented as fold change +/- SEM.

Figure 4. APP-like immunoreactivity at various time points post-FPI. APP staining was observed 24 hours post injury at the cortical injury site (a), particularly in cell bodies and axons (black arrows) and within the ipsilateral hippocampal formation (b). APP immunoreactvity remained strong in the injured cortex (c) and hippocampus (d) at 7 days post-injury (c). Scale bar=100um.

Figure 5. Tau reactivity in hippocampus post-FPI. Tau reactivity was increased in neurons within the injured cortex and hippocampus. Cortical staining was particularly intense within cell bodies 3 days post-injury (a) (black arrows). Tau staining gradually increased with time post-FPI in contralateral hippocampus and was strongest 7 days post-injury, particularly in cell bodies (b). Scale bar=100 $\mu \mathrm{m}$. 
Table 1. Summary of qualitative protein levels at 24 hours, 3 days and 7 days post-FPI (relative to sham operated controls).

\begin{tabular}{llllll}
\hline Time point & Area & APP & BACE-1 & Nct & Tau \\
\hline \multirow{7}{*}{ hours } & ipsi. CX & ++ & + & ++ & + \\
& ipsi.hip & ++ & ++ & + & ++ \\
& contra. cx & - & + & - & - \\
\multirow{5}{*}{3 days } & contra. Hip & - & - & - & - \\
& ipsi. cx & +++ & ++ & - & ++ \\
& ipsi. hip & + & + & - & +++ \\
& contra.cx & - & + & - & - \\
& contra. hip & - & - & - & + \\
days & ipsi. cx & ++ & - & ++ & ++ \\
& ipsi. hip & ++ & - & + & ++ \\
& contra. cx & - & - & + & - \\
& contra. hip & - & - & - & ++ \\
\hline
\end{tabular}

(No change $=-,+$ mild increase, +++ , most intense. APP $=$ amyloid precursor protein, Nct $=$ nicastrin, ipsi $=$ ipsilateral to injury, contra $=$ contralateral to injury). 

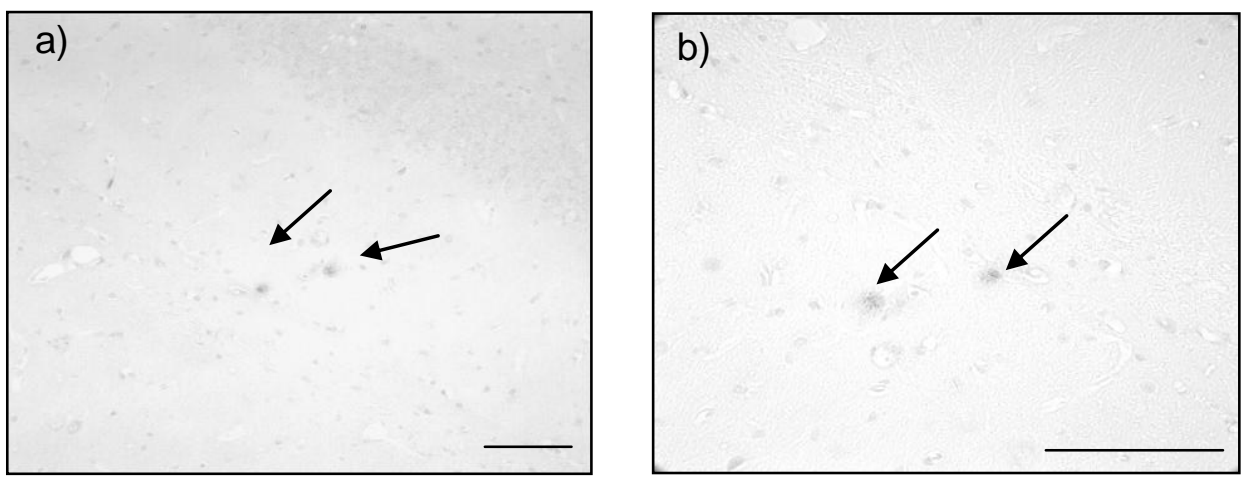

c) Temporal cortex

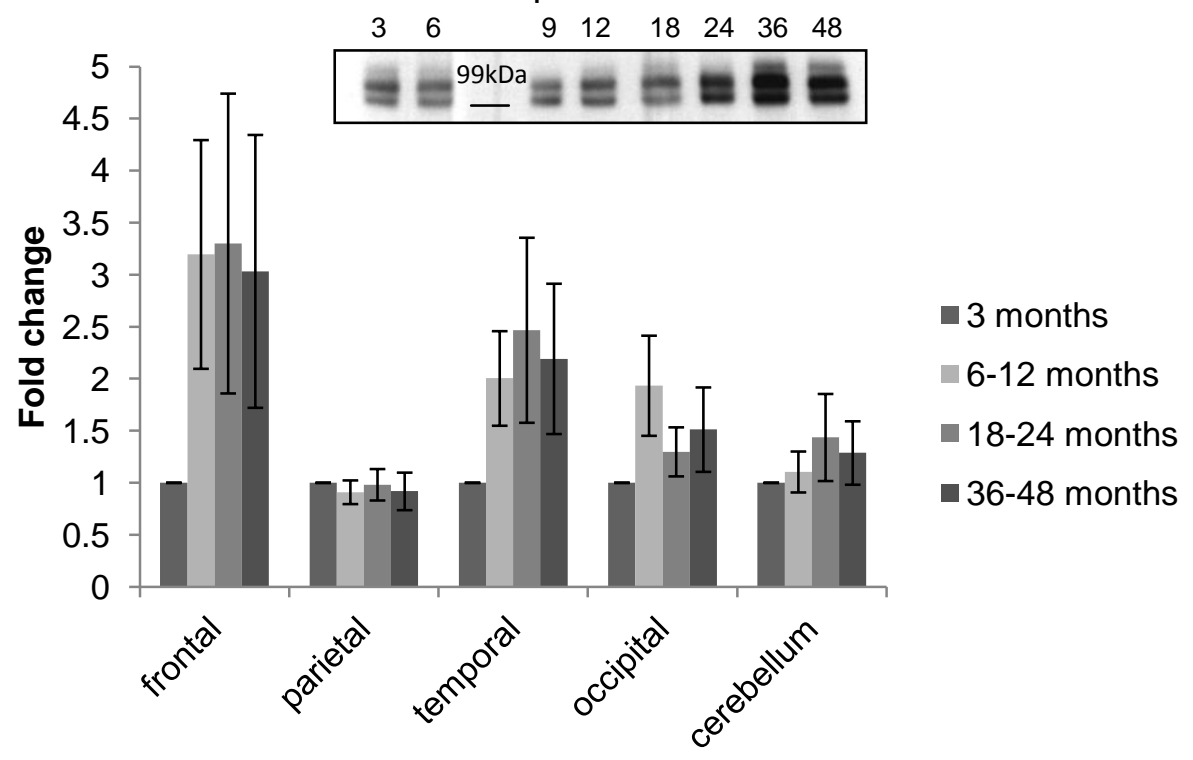

d) Hippocampus
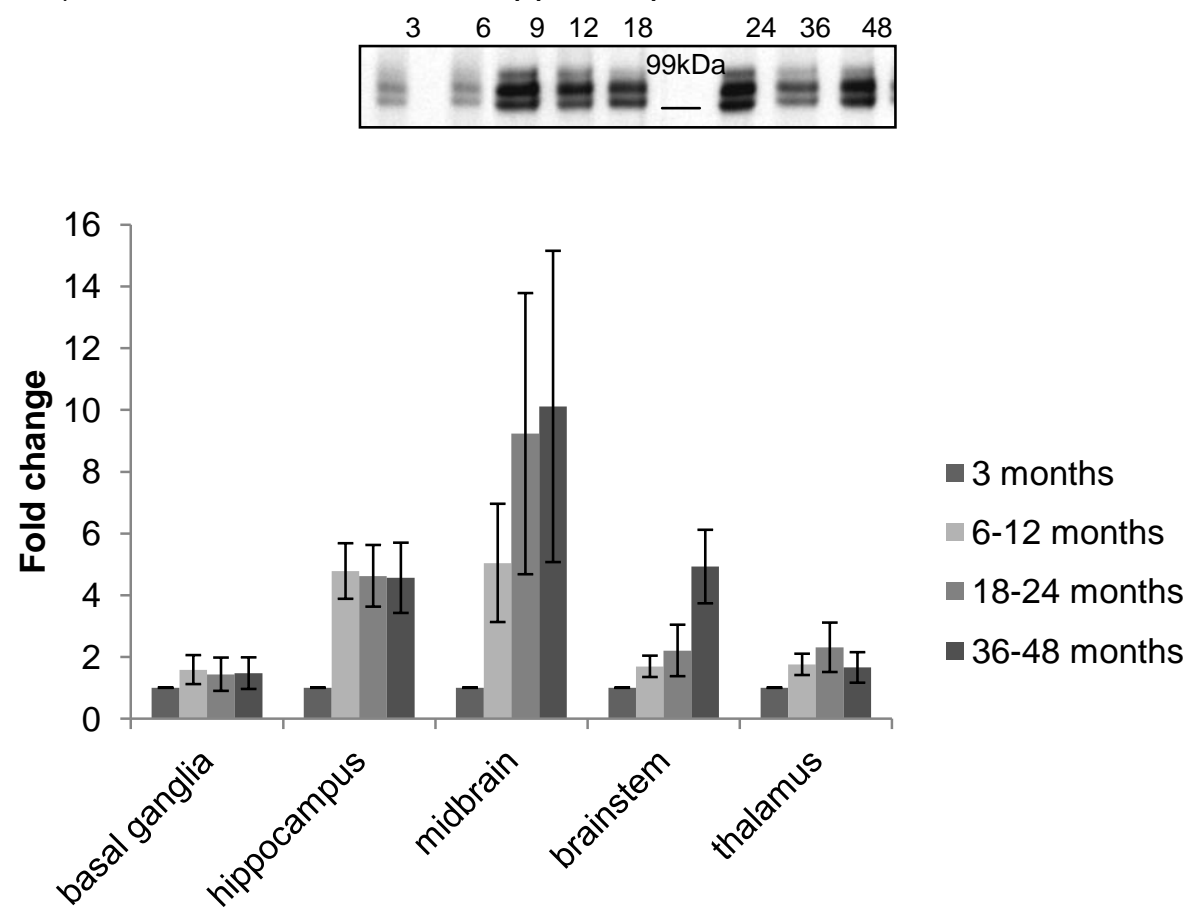
Figure 2

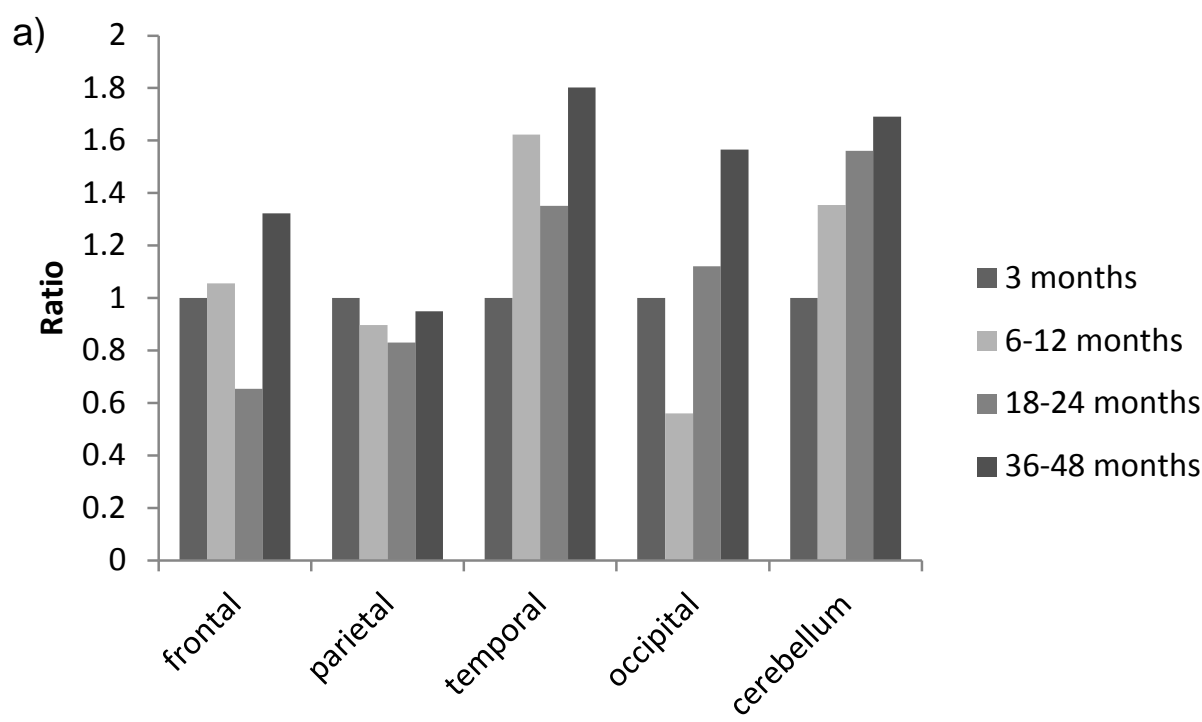

b)

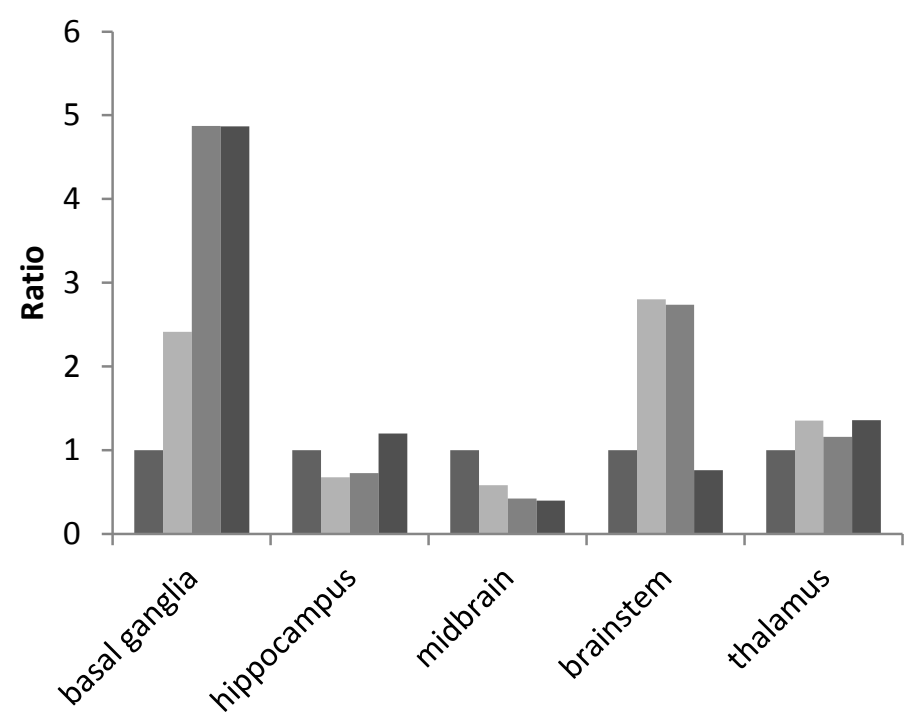

- 3 months

6-12 months

a 18-24 months

- 36-48 months 

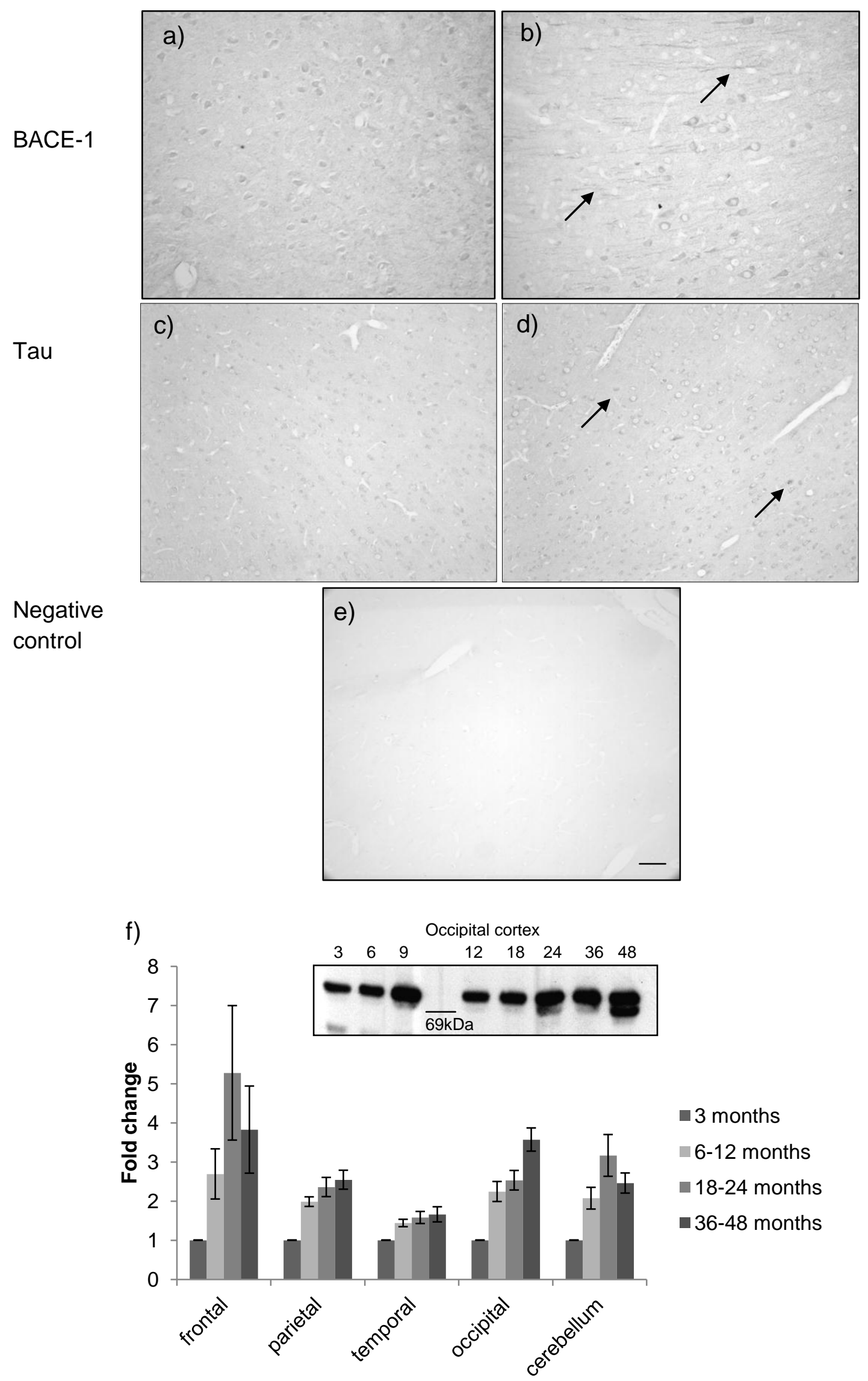


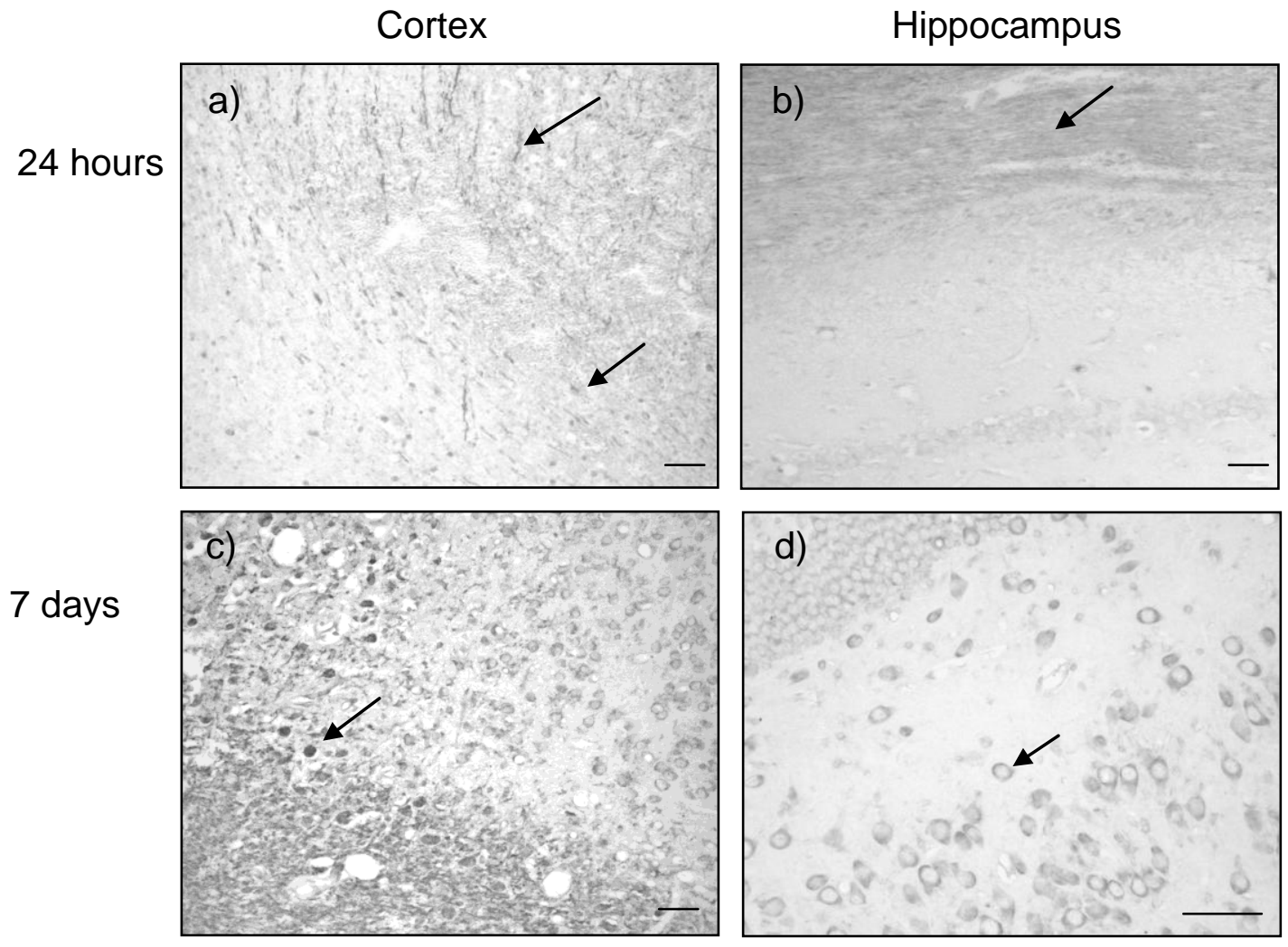



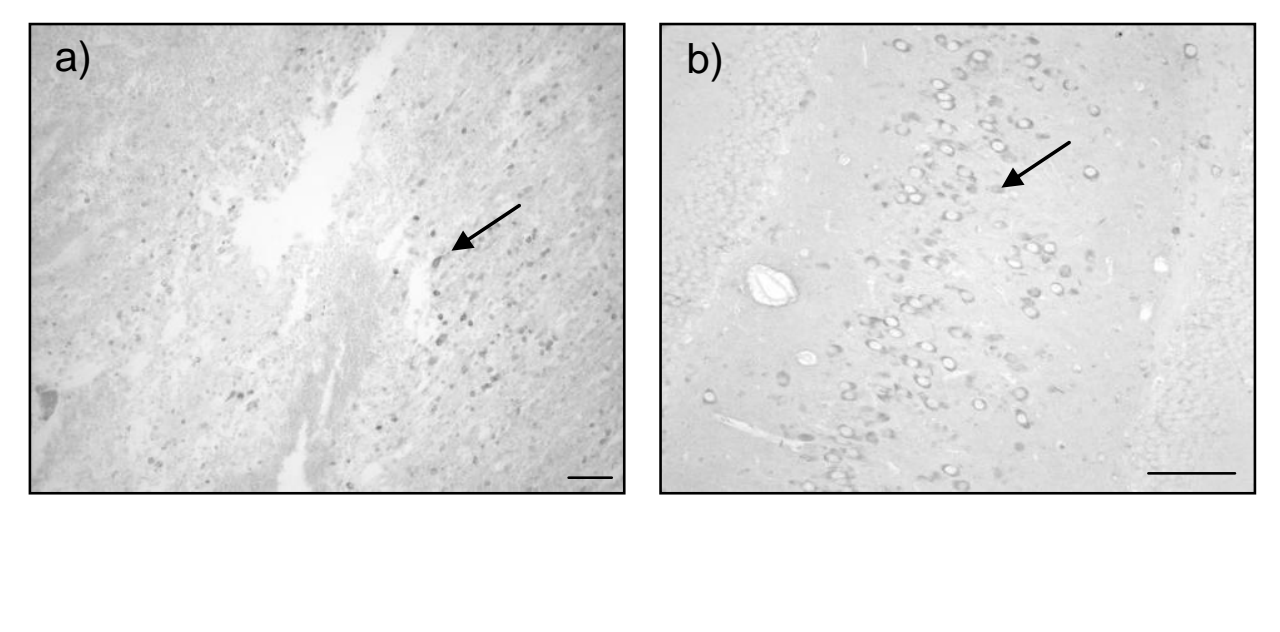

Figure 5

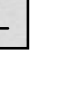

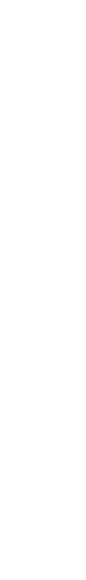

\section{Figure 5}

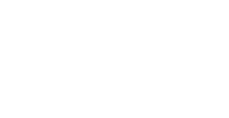

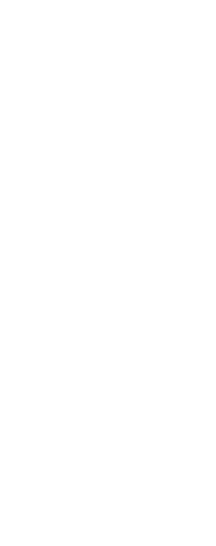
(2)
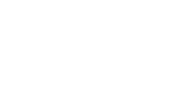